\title{
Aneurisma gigante de la arteria esplénica. Reporte de un caso
}

\author{
Luis Hernández M. ${ }^{1,2}$, Alejandro Kotlik A., \\ Juan Bombin F. ${ }^{1,2}$ y Jaime Recasens T.,2
}

Servicio de Cirugía. Hospita Dr. Eduardo Pereira Ramírez. Valparaíso, Chile. Departamento de Cirugía. Universidad de Valparaíso. Valparaíso, Chile.

Recibido el 29 de septiembre de 2019 y aceptado para publicación el 18 de noviembre de 2019 .

Correspondencia a: Dr. Luis Hernández M. luiscesarh@gmail.com

\section{Giant splenic artery aneurysm. Case report}

Aim: To report the case of a giant splenic artery aneurysm of $9.5 \mathrm{~cm}$ diameter, in a 75 years old woman, who consulted for vertiginous syndrome, with incidental finding to the physical examination of pulsatile abdominal mass, confirmed by angiotomography of abdomen and pelvis. Materials and Method: Clinical record of a patient undergoing open surgical repair of a giant splenic aneurysm. Results: By laparotomy, anterior approach, the ligation of the aneurysm is performed with partial resection of the aneurysm sac plus splenectomy, evolving without complication at 12 months of follow-up. Discussion: The splenic artery aneurysm, is defined at any dilatation greater than $1 \mathrm{~cm}$ in diameter, it is surgically indicated when they measure more than $2 \mathrm{~cm}$ and called giant from $5 \mathrm{~cm}$. Conclusion: Splenic artery aneurysm is a uncommon condition, usually asymptomatic, but potentially fatal especially in pregnant women, in the literature there are only 78 reported cases of giant splenic artery aneurysms, expedited surgical resolution is recommended.

Key words: giant splenic artery aneurysm; huge splenic artery aneurysm; visceral artery aneurysm.

\section{Resumen}

Objetivo: Reportar el caso de un aneurisma gigante de la arteria esplénica de $9,5 \mathrm{~cm}$ de diámetro, en una mujer de 75 años de edad, que consultó por síndrome vertiginoso, con hallazgo incidental al examen físico de masa abdominal pulsátil, confirmado mediante angiotomografía de abdomen y pelvis. Materiales y Método: Registro clínico de una paciente sometida a reparación quirúrgica abierta de una aneurisma gigante de la arteria esplénica. Resultados: Mediante laparotomía, abordaje anterior, se efectúa la ligadura del aneurisma con resección parcial del saco aneurismático más esplenectomía, evolucionando sin complicación a 12 meses de seguimiento. Discusión: Se define como aneurisma de la arteria esplénica a toda dilatación mayor a $1 \mathrm{~cm}$ de diámetro, presenta indicación quirúrgica cuando mide más de $2 \mathrm{~cm}$ y se denomina gigante a partir de los $5 \mathrm{~cm}$. Conclusión: Los aneurismas de la arteria esplénica son una condición infrecuente, habitualmente asintomática, pero potencialmente mortal especialmente en embarazadas; en la literatura solo existen 78 casos reportados de aneurismas gigantes de la arteria esplénica, se recomienda la resolución quirúrgica expedita.

Palabras clave: aneurisma gigante de la arteria esplénica; aneurisma de la arteria esplénica enorme; aneurisma de las arterias viscerales.

\section{Introducción}

El aneurisma de la arteria esplénica (AAE) es el más frecuente entre los aneurismas viscerales, representando el $60 \%$ de los mismos ${ }^{1,2}$ y el tercero en frecuencia después de los aórticos e ilíacos ${ }^{1-3}$. Se define como AAE a toda dilatación mayor a $1 \mathrm{~cm}$ de diámetro, presenta indicación quirúrgica cuando mide más de $2 \mathrm{~cm}$ y se denomina gigante a partir de los $5 \mathrm{~cm}^{2,3}$.

La revisión de estudios angiográficos indica una incidencia estimada del $0,8 \%$ en la población general $^{4,5}$, la mayoría de los AAE verdaderos son saculares, menores a $2 \mathrm{~cm}$ de diámetro y se presen- 
tan en el tercio distal (75\%), seguidos por el tercio medio $(20 \%)^{6}$, con predominio entre los 50 a 70 años de edad $(70 \%)^{5,6}$.

La patogénesis de los aneurismas viscerales se considera secundaria a arteriosclerosis (32\%), degeneración/displasia (24\%), traumatismos abdominales $(22 \%)$, infección o enfermedad inflamatoria $(10 \%)$, alteraciones del tejido conectivo (síndrome de Marfan, síndrome de Ehler Danlos, displasia fibromuscular, síndrome de Kawasaki), situaciones de hiperflujo como el embarazo y la hipertensión portal $^{2-4}$. En la literatura solo existen 78 casos reportados de aneurismas gigantes de la arteria
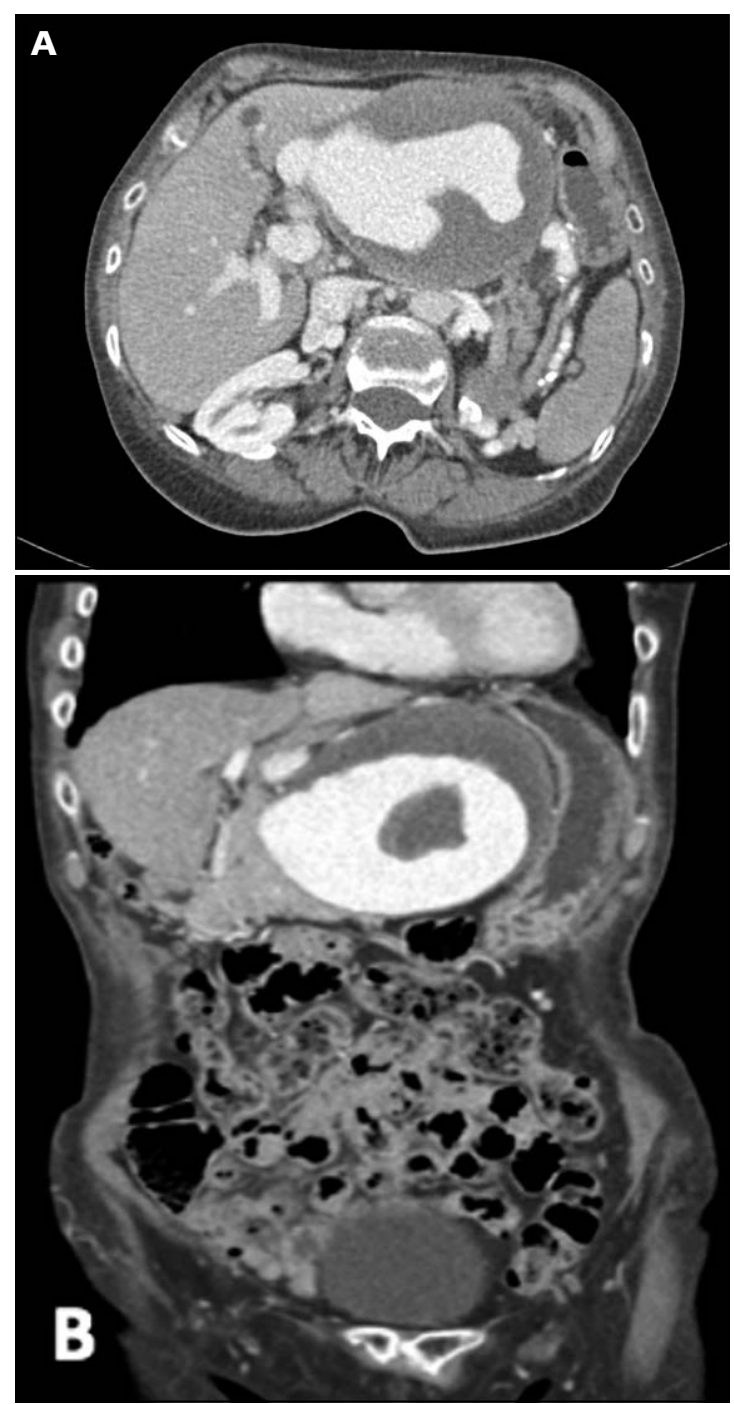

Figura 1. Angiotomografía de abdomen y pelvis. A: (corte transversal) y B: (corte coronal), evidencian aneurisma parcialmente trombosado, del tercio distal de la arteria esplénica, de $95 \times 80 \times 60 \mathrm{~mm}$. esplénica, comunicamos el caso de una paciente tratada en nuestro servicio.

\section{Caso clínico}

Paciente de sexo femenino de 75 años de edad, sin comorbilidades asociadas, quien consulta por presentar síndrome vertiginoso agudo, con hallazgo al examen físico de masa abdominal pulsátil, se realiza ultrasonido abdominal, el cual reporta imagen de posible quiste hidatídico, que ocupa el lóbulo hepático izquierdo de $9,8 \mathrm{~cm}$, razón por la cual se indica angiotomografía de abdomen y pelvis, evidenciando aneurisma gigante bilobulado de la arteria esplénica distal, de 9,5 × 8 × $6 \mathrm{~cm}$, con calcificaciones parietales, ubicado entre el páncreas y estómago, el cual produce compresión extrínseca de la vena esplénica, ocasionando hipertensión portal leve. A su vez se evidencia aneurisma de $1 \mathrm{~cm}$ de diámetro, del tercio medio de la arteria renal derecha y la arteria hepática común de origen en la arteria mesentérica superior (Figuras 1, 2 y 3).

Al examen físico se evidencia aumento de volumen de $10 \times 8 \mathrm{~cm}$, localizado en el hemiabdomen izquierdo, pulsátil, con soplo, sin frémito, superficie lisa, adherido a planos profundos, no doloroso. Exámenes preoperatorios sin alteración, riesgo anestésico quirúrgico ASA II, GOLDMAN I.

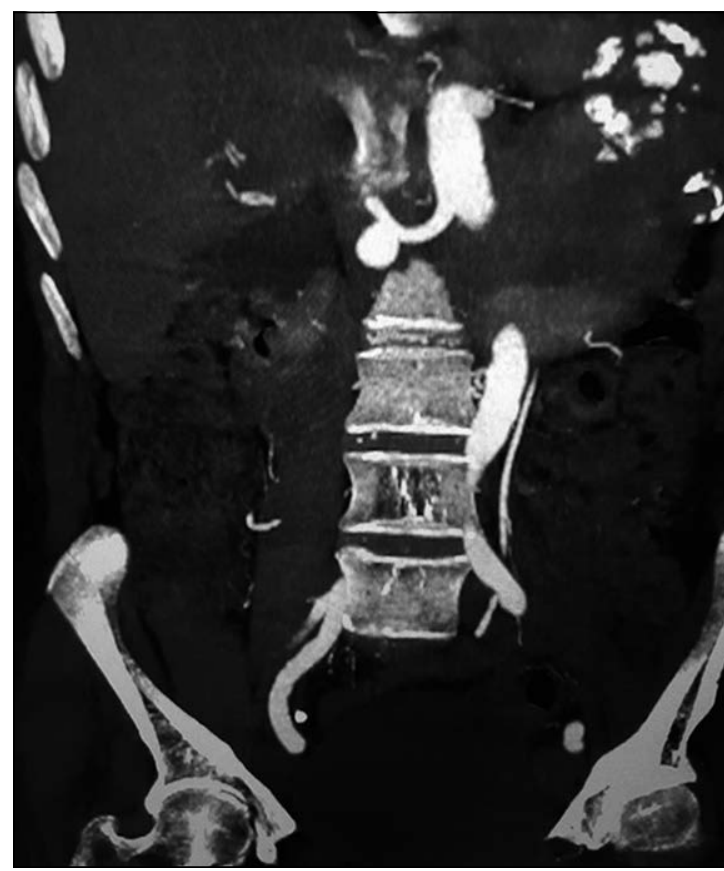

Figura 2. Angiotomografía de abdomen y pelvis (corte coronal), se evidencia aneurisma de $10 \mathrm{~mm}$ de diámetro de la arteria renal derecha. 
Mediante laparotomía, abordaje anterior, línea media, se ingresa a la transcavidad de los epiplones y evidencia aneurisma de la arteria esplénica distal de $9,5 \times 8 \mathrm{~cm}$, firmemente adherido a la cara anterior del páncreas. En vista de hallazgos, se realiza ligadura proximal y distal del mismo, apertura del saco aneurismático, retiro de trombos intramurales, ligadura transfixiante de arterias afluentes, resección parcial del saco aneurismático y cierre del saco remanente con Poliglactina 910, ameritando esplenectomía total debido a isquemia esplénica irreversible (Figura 4).

Figura 3. Reconstrucción tridimensional de la aorta abdominal, se evidencia aneurisma esplénico (sombreado), flecha señala aneurisma de la arteria renal derecha.
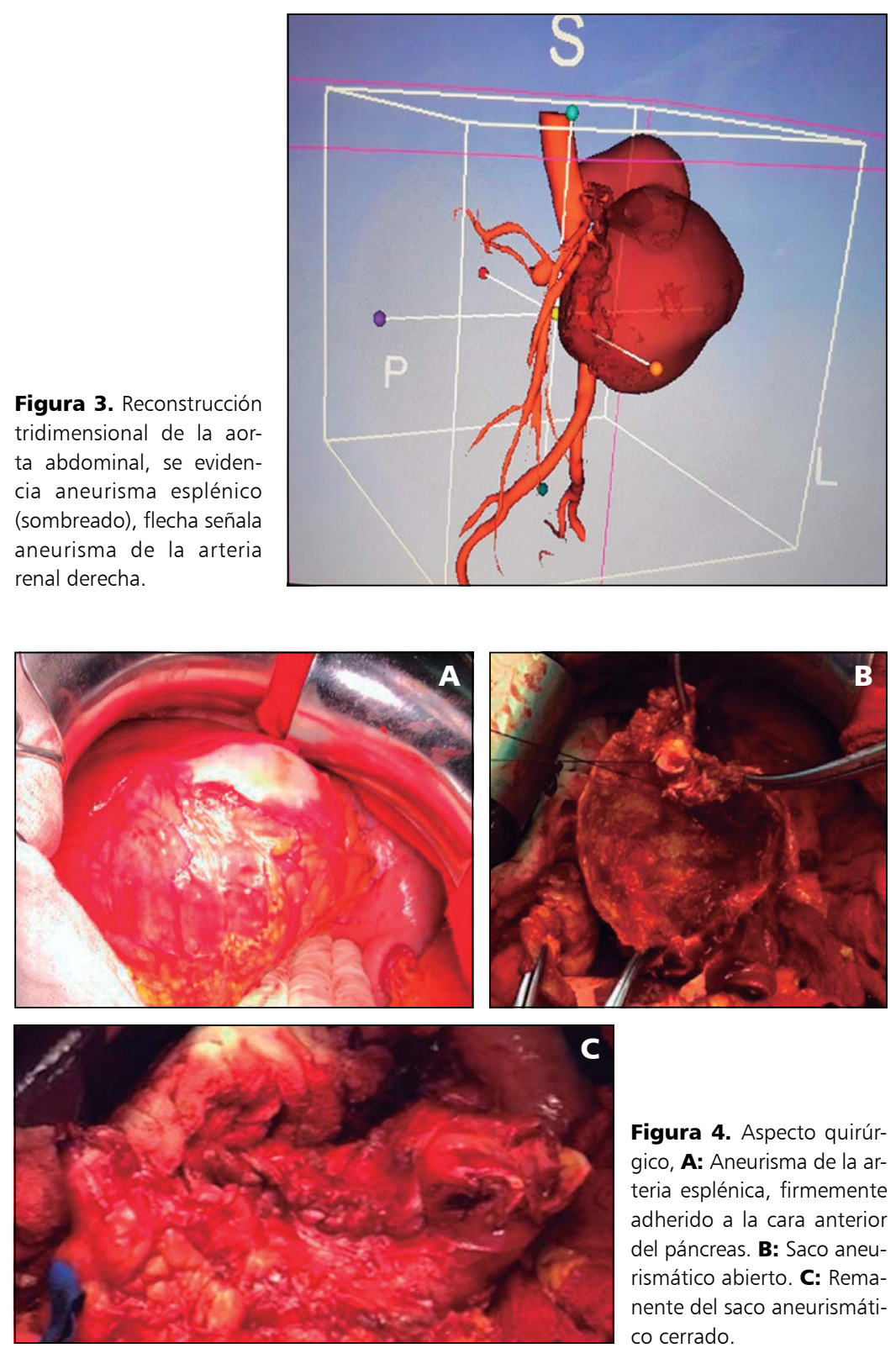

Figura 4. Aspecto quirúrgico, A: Aneurisma de la arteria esplénica, firmemente adherido a la cara anterior del páncreas. B: Saco aneurismático abierto. C: Remanente del saco aneurismático cerrado.
Procedimiento quirúrgico sin otros incidentes, con sangrado aproximado de $300 \mathrm{cc}$, se utiliza instrumental convencional para laparotomía y pinza bipolar (LigaSure ${ }^{\mathrm{TM}}$ ). La paciente presenta elevación transitoria leve de las enzimas pancreáticas, normalizándose a las $72 \mathrm{~h}$ del procedimiento, egresando de la institución a los 6 días tras la cirugía, encontrándose estable, sin falla de órganos, con leve dolor y exámenes de sangre normales, recibiendo vacunación profiláctica contra infección por gérmenes encapsulados a los 14 días del postoperatorio.

Control durante 12 meses, paciente sin complicaciones, autovalente, desarrollando sus actividades habituales. Angiotomografía de control al mes y 12 meses, sin evidencia de captación de contraste en el remanente aneurismático y aneurisma renal sin alteración en su diámetro, sin complicaciones.

\section{Discusión}

Los AAE predominan en mujeres en una relación de $4: 1^{2,6,7}$, detectándose especialmente en multíparas (80\%), aproximadamente el $80 \%$ cursan de forma asintomática $^{2}$, siendo identificados incidentalmente en estudios de imagen ${ }^{7}$. En algunos casos se presentan con sintomatología abdominal inespecífica, como aumento de volumen o dolor en el hipocondrio y/o flanco izquierdo, que se irradia a la escápula ipsilateral ${ }^{2,3}$. Pueden presentar embolia, trombosis, compresión o fistulizar a estructuras vecinas, como tubo digestivo o páncreas, produciendo hemorragia digestiva o hemosuccus pancreaticus respectivamente $^{8,9}$.

El riesgo de rotura, se encuentra entre el 2\%$10 \%$, llegando al $70 \%$ en pacientes embarazadas, con una mortalidad fetal cercana a $95 \%{ }^{8}$, a predominio en el tercer trimestre del embarazo, presentando síntomas que se pueden confundir con otras emergencias obstétricas.

La rotura de los AAE en el 25\% de los casos, es contenida en el retroperitoneo durante varias horas, hasta producirse finalmente inestabilidad hemodinámica (fenómeno de la "doble rotura") 8,9 La mortalidad global es de 25\%-75\%, mientras que la mortalidad debido a la cirugía reglada es de $0,5 \%-1,5 \%{ }^{10,11}$.

Los pseudoaneurismas de la arteria esplénica son menos prevalentes, con predominio en el sexo masculino 2:1 y están en relación con historia de pancreatitis, traumatismo o iatrogenia ${ }^{6}$. En los casos relacionados con pancreatitis, generalmente se trata de un proceso crónico que incluye la presencia de pseudoquistes pancreáticos, son casos con mal pronóstico, elevado riesgo de sangrado digestivo (50\%) 
y mortalidad cercana al 50\% ${ }^{1,6}$. Estos pueden presentar sangrado intermitente dentro del quiste pancreático, ducto pancreático (hemossucus pancreaticus) o al duodeno ${ }^{8,9}$, razón por la cual presentan indicación quirúrgica independientemente del tamaño ${ }^{8}$.

Se sugiere el tratamiento quirúrgico en pacientes con aneurismas mayores a $2 \mathrm{~cm}$ de diámetro, siempre valorando que la intervención electiva presente una mortalidad inferior al $1,5 \%{ }^{7}$. Se considera el tratamiento quirúrgico en aneurismas menores de $2 \mathrm{~cm}$, en caso de crecimiento rápido mayor a $5 \mathrm{~mm} / a n ̃ o$, trasplante hepático, embarazadas, pacientes en edad reproductiva, hipertensión portal, enfermedad severa del tejido conectivo y en pseudoaneurismas ${ }^{7}$.

En pacientes sin factores de riesgo con aneurismas menores a $2 \mathrm{~cm}$, se sugiere control anual con ultrasonido abdominal en manos de operadores expertos. En series de seguimiento, se ha descrito la necesidad de tratamiento quirúrgico en un $10 \%$ de los pacientes controlados ${ }^{2,6}$.

Para los AAE las opciones de tratamiento son la cirugía abierta, laparoscópica, endovascular y el manejo conservador, este último basado en el seguimiento y control de factores de riesgo asociados. La cirugía abierta presenta una tasa de complicaciones cercana al $9 \%$, con una mortalidad asociada del $1,3 \%{ }^{12}$, el abordaje es por vía retroperitoneal (anterior o lateral), siendo posible realizar la remoción completa del aneurisma, la ligadura proximal y distal de la arteria esplénica con resección total o parcial del aneurisma (como en nuestra paciente) o la ligadura transaneurismática.

La resección electiva de los aneurismas esplénicos, amerita esplenectomía en un $29 \%$ de los casos debido a infarto esplénico ${ }^{10}$. La técnica laparoscópica, se sugiere para aneurismas menores a $5 \mathrm{~cm}$ y aneurismas pequeños en embarazadas, preferiblemente en el $2^{\circ}$ trimestre y antes de las 20 semanas de gestación, requiere de cirujanos expertos y ecografía intraoperatoria ${ }^{12}$.

La embolización transcatéter se sugiere para aneurismas menores a $5 \mathrm{~cm}$ y posee tasas de éxito que oscilan entre el 55 al $100 \%{ }^{11,12}$, se prefiere en los casos de elevada complejidad quirúrgica, elevado riesgo quirúrgico y lesiones localizadas en el hilio esplénico. Las complicaciones más frecuentes son la migración del agente embolizante, rotura del aneurisma, infarto intestinal, infarto esplénico y abscesos. $^{12}$.

En una revisión de la literatura global realizada en el $2015^{13}$, la cual incluyó 69 artículos sobre AEE gigantes, con una suma de 78 pacientes $(64 \%$ hombres, $36 \%$ mujeres), la edad media para el sexo masculino fue de 57,5 años y para el femenino 52,7 años, de estos presentaron síntomas el 70,4\% y asintomáticos diagnosticados incidentalmente el $29,6 \%$.

Los factores predisponentes más frecuentes fueron la pancreatitis aguda o crónica, aterosclerosis, hipertensión portal y cirrosis hepática. Las dimensiones del aneurisma se obtuvieron con un rango de 5 a $30 \mathrm{~cm}$ (media 9,7 cm) ${ }^{13}$.

La complicación más frecuente de los AAE gigantes, fue la rotura en un 19,2\%, todas en aneurismas mayores a $10 \mathrm{~cm}$, con una mortalidad por rotura del 33,3\% y mortalidad en el postoperatorio temprano del 10,2\%, presentado predominio en el sexo masculino (73,3\%). El resto de las complicaciones fueron del tipo fístula: gastroesplénica $(3,8 \%)$, arteriovenosa esplénica $(3,8 \%)$, coloesplénica $(1,2 \%)$, pancreática $(1,2 \%)$ y portoesplénica $(1,2 \%)^{13}$.

En relación al aneurisma de la arteria renal (AAR) de $1 \mathrm{~cm}$, que presenta nuestra paciente, hasta la presenta fecha no ha modificado sus dimensiones, manteniéndose en control periódico ambulatorio con ultrasonido abdominal.

Los AAR presentan una incidencia del 0,7 al $1,3 \%$, su desarrollo se ha relacionado con múltiples mecanismos, entre ellos displasia fibromuscular, arteriosclerosis, alteraciones congénitas, vasculopatías inflamatorias y traumatismos. Generalmente son asintomáticos, pudiendo producir dolor en flanco o subcostal (15\%), hematuria (30\%), HTA $(55 \%)^{14}$.

Se describen cuatro tipos diferentes de AAR, saculares $(85 \%)$, fusiformes, asociados a disección e intrarenales ${ }^{14}$. Las indicaciones quirúrgicas en AAR son tamaño mayor a $2 \mathrm{~cm}$, pacientes monorenales, hipertensión arterial de difícil manejo, calcificación anular incompleta, mujer en edad fértil, aumento progresivo de tamaño, fístula arteriovenosa y complicaciones asociadas (hematuria, infarto renal, ruptura $)^{14}$.

El resto de los pacientes pueden ser manejados de forma conservadora, aunque algunos autores sugieren la reparación en todos los casos, independientemente del tamaño o síntomas, con la finalidad de evitar microembolismos al parénquima renal, o el desarrollo de hipertensión mantenida ${ }^{14}$.

Las opciones quirúrgicas incluyen embolización, resección del aneurisma y anastomosis directa, resección y reparación con parche de Dacrón, autotransplante tras resección del aneurisma, cirugía ex vivo con autotransplante, stent intravascular por vía percutánea, nefrectomía parcial o total. ${ }^{14}$

En relación a la variante anatómica en nuestra paciente, de la arteria hepática común de origen en la 
mesentérica superior, este hallazgo se presenta con una frecuencia del 1,5 al 2,5\% ${ }^{15}$, siendo el origen más común en el tronco celíaco en un 55 al $75 \%$ de los $\operatorname{casos}^{15}$.

\section{Conclusión}

Los aneurismas esplénicos gigantes, aunque infrecuentes, representan una patología potencialmente mortal, se recomienda su estudio oportuno y resolución quirúrgica expedita.

\section{Responsabilidades éticas}

Protección de personas y animales. Los autores declaran que para esta investigación no se han realizado experimentos en seres humanos ni en animales.

Confidencialidad de los datos. Los autores declaran que en este artículo no aparecen datos de pacientes.

Conflictos de interés: no hay.

\section{Bibliografía}

1. Busuttil RW, Gelabert HA. Visceral Artery aneurysms. En: Haimovici H, editor. Vascular surgery. 4th ed Massachusetts: Blackwell Science Inc; 1996. p. 842-52.

2. Rockman CB, Maldonado TS. Splanchnic artery aneurysms. En: Johnston CA, editor. Rutherford's vascular surgery. 7th ed Philadelphia: Saunders Elsevier; 2010. p. 2140-55.

3. Barreiro-Mouro A, Alonso-Pérez M, Adeba Vallina E. Aneurismas viscerales. En: Morillo FV, editor. Tratado de las enfermedades vasculares. Barcelona: Viguera editores; 2006. p. 827-41.

4. Williams RA, Mueller M, Wilson SE. Splanchnic artery aneurysms. En: Veith FJ, editor. Vascular surgery principles and practice. 2nd ed New York: McGraw-Hill, Inc; 1994. p. 580-8.

5. Lakin RO, Bena JF, Sarac TP, Shah S, Krajewski LP, Srivastava SD, et al. The contemporary management of splenic artery aneurysms. J Vasc Surg. 2011;53:958-65

6. Berceli SA. Hepatic and splenic artery aneurysms. Semin Vasc Surg. 2005; 18:196-201.

7. Hogendoorn W, Lavida A, Hunink M, Moll FL, Geroulakos G, Muhs BE, et al. Open repair, endovascular repair, and conservative management of true splenic artery aneurysms. J Vasc Surg. 2014;60:1667-76.

8. Deshpande, Kulkarni VM, Rege S, Dalvi AN, Hardikar JV. Ruptured true aneurysm of the splenic artery: an unusual cause of hemoperitoneum. J Postgrad Med. 2000;46:191-2.

9. Wagner WH, Cossman DV, Treiman RL, Foran RF, Levin PM, Cohen JL. Hemosuccus pancreaticus from intraductal rupture of a primary splenic artery aneurysm. J Vasc Surg. 1994;19:158-64.

10. Abad C, Montesdeoca Cabrera D, Sáez-Guzmán T. Aneurisma de la arteria esplénica. Revisión de dos casos intervenidos quirúrgicamente. An Med Interna 2006;23:130-2

11. Pulli R, Dorigo W, Troisi N, Pratesi G, Innocenti AA, Pratesi C. Surgical treatment of visceral artery aneurysms: A 25 year experience. J Vasc Surg. 2008;48:334-42.

12. Marone EM, Mascia D, Kahlberg A, Brioschi C, Tshomba Y, Chiesa R. Is open repair still the gold standard in visceral artery aneurysm management? Ann Vasc Surg. 2011;25:936-46.

13. Akbulut S, Otan E. Management of Giant Splenic Artery Aneurysm: Comprehensive Literature Review. Medicine (Baltimore). 2015;94(27):e1016.

14. Wayne EJ, Edwards MS, Stafford JM, Hansen KJ, Corriere MA. Anatomic characteristics and natural history of renal artery aneurysms during longitudinal imaging surveillance. J Vasc Surg. 2014;60:448-53.

15. Hiatt J, Gabbay J, Busuttil R. Surgical anatomy of the hepatic arteries in 1000 cases. Ann Surg. 1994;220:50-2. 